\title{
Going through the Motions: A Time-and- Motion Study of Workload Associated with Nonformulary Medication Orders
}

\author{
Elaine Chang, Angus Kinkade, Anthony C H Tung, and Aaron M Tejani
}

\section{INTRODUCTION}

$\mathrm{H}$ ospitals organize medications according to a formulary system to guide appropriate medication use. ${ }^{1}$ Medications approved on formulary have been assessed by a pharmacy and therapeutics committee and are provided in hospitals because of advantages in safety and efficacy, or because of cost savings (when safety and efficacy are equivalent to the safety and efficacy of alternative medications). Formulary medications are stocked in the pharmacy and may be ordered for approved indications. Nonformulary (NF) medications have not been formally assessed or there is no evidence suggesting that they have greater therapeutic value than formulary alternatives. ${ }^{1} \mathrm{NF}$ medication orders must be reviewed on a case-by-case basis before the medication is dispensed.

The Lower Mainland Pharmacy Services in British Columbia has an algorithm for assessing NF medication orders (Appendix 1, available at www.cjhp-online.ca/index.php/ cjhp/issue/view/120/showToc). ${ }^{2}$ This assessment determines whether there is an automatic therapeutic interchange policy to switch the medication to a formulary alternative without consulting the original prescriber. If no automatic interchange is possible, a pharmacist will determine whether the medication can be discontinued, switched to an alternative formulary medication after discussion with the prescriber, or dispensed using the patient's own medication. If none of those options are available, the NF medication should be purchased into the hospital pharmacy if necessary and the order dispensed using the hospital's supply.

In Lower Mainland Pharmacy Services, decisions about whether a medication should be considered for addition to the formulary have not historically considered the cost of assessing each NF order. Typically when NF medications are reviewed for inclusion on the formulary, their efficacy and safety are compared with the efficacy and safety of alternative therapies. When best available evidence suggests similar therapeutic activity, then the acquisition costs of the 2 drugs are compared. Without considering the time required to assess each NF medication order, formulary committees may leave medications off formulary on the basis of a cost minimization analysis of acquisition cost alone, which has the effect of increasing hidden labour costs.

Previous studies have evaluated the time required by pharmacy staff to assess and process NF medication orders. In one previous study, pharmacy personnel estimated the frequency of NF medication orders as $3.9 \%$ to $19.4 \%$ of all orders, with the average total time spent on NF medication orders between 4 and $69 \mathrm{~min}$, depending on how the order was assessed and processed. ${ }^{3}$ The least labour-intensive scenario involved switching an order for an NF medication to a therapeutically equivalent drug. The scenario that required the most time to process was when the medication was to be supplied by the hospital and had to be ordered from a supplier and repackaged. Although the study by Helmon and others ${ }^{3}$ presents valuable information, the data were obtained through interviews rather than objective measurements, which may have introduced recollection bias.

Another study compared the cost impact of using a patient's own medication for routine hospital dispensing. ${ }^{4}$ The average time required to verify the supply of a patient's own medication was $4.8 \mathrm{~min}$ per medication. The outcome for this study was the drug acquisition cost, as the medications evaluated were readily available and labour time was negligible. Using patients' own medications resulted in cost savings of $74 \%$ relative to dispensing through the hospital, primarily through reduction of drug wastage in multidose inhalers. However, that study did not examine the labour times and costs required for NF medication orders dispensed through the hospital. Further- 
more, time was recorded only when the pharmacist arrived on the patient care unit, whereas it may be important to also consider the pharmacist's work taking place in the dispensary when accounting for total labour costs.

The pharmacy staff at St Paul's Hospital (SPH) follows the Lower Mainland Pharmacy Services algorithm for assessing NF orders (Appendix 1). SPH is a 400-bed acute care hospital in Vancouver, British Columbia, ${ }^{5}$ and was chosen for this evaluation because high compliance with the policy and a high rate of orders for NF medications were anticipated to allow for better data collection.

The primary objective of this study was to determine the amount of time spent on a typical NF medication order at $\mathrm{SPH}$. The secondary objectives were to determine how many NF medication orders are received in a given day and the outcomes of those orders. Data were gathered through the use of both objective time measurements and self-reported times from pharmacists. The results obtained from this study will be used to better inform formulary decisions by incorporating the labour time for NF assessments into the accounting of total costs for decision alternatives.

\section{METHODS}

This study measured pharmacists' activity during the assessment and processing of NF orders. All relevant steps in the NF assessment process at $\mathrm{SPH}$ were mapped out, and time was measured for each task.

The primary outcomes of this evaluation were the number of NF orders passing through each path of the NF assessment algorithm and the average time required for each path: automatically substituting a formulary drug, switching to a therapeutically equivalent alternative after discussion with a prescriber, discontinuing the order, verifying patients' own medications, or dispensing using hospital supply. Secondary outcomes included the average number of NF medication orders per day, the average time required to assess and process a typical NF medication order, and the average time required to assess and process only those NF medication orders that were dispensed using patients' own medications or the hospital supply.

The study included all orders involving NF medications processed through all possible paths of the assessment algorithm; no orders were excluded. Data were collected over the period June 1 to August 24, 2015, at SPH, on 3 or 4 days each week, selected to include similar proportions of day and evening shifts across all days of the week.

Dispensary pharmacists were directly observed and timed on specific tasks pertaining to NF medication orders. Data collection relied on dispensary pharmacists indicating that they were about to begin working on an NF medication order, at which point a timer was started. The timer was stopped when the task was completed, and the time was recorded. Only time spent by staff on tasks related to NF orders was recorded. For example, if a pharmacist received an urgent task while working on an NF medication order, and had to set aside the NF order, the timer was paused at the moment they stopped working and was restarted when they returned to the NF order.

The relevant steps in supplying an NF medication by using a patient's own medication included the dispensary pharmacist assessing the order, paging and communicating with the clinical pharmacist, repackaging the medication, and filling out a form to document the NF medication order assessment. The relevant steps in providing an NF medication through hospital supply included the dispensary pharmacist assessing the order, paging and communicating with the clinical pharmacist, obtaining approval from the dispensary supervisor, and entering the new order. The clinical pharmacists documented their own times for tasks relating to NF medication order assessments, including communication by phone with the dispensary pharmacist; discussion with patients, their family or friends, or the original prescribers; and repackaging of the medication. In the evenings, NF medication orders that could not be automatically substituted were designated as "medication not available" and were evaluated the next morning by pharmacy staff members when they were available on the patient care wards. Medication orders for noncritical medications were put on hold or discontinued after discussion with the health care team.

To determine the total number of NF medication orders received in a day, data collection forms were placed at each workstation on the designated data collection days. Dispensary pharmacists were asked to record each NF medication order received by writing the medication name and indicating the outcome, except for medications automatically interchanged to formulary alternatives (automatic substitutions are filed daily, so the total values could be obtained through direct observation of records).

Tasks that were not observed, such as medication purchase, were not recorded, as these are routine components of inventory management.

A presentation was given to the clinical pharmacists to outline the purpose and methodology of the project. Clinical pharmacists were asked to document the time that they spent specifically on tasks pertaining to NF orders, including those for medications that were switched to a formulary alternative, supplied with patients' own medications, or supplied and dispensed by the hospital. The pharmacists were given standardized data collection forms containing tables with column headings "date", "drug name", "time spent", and "method of resolution". They continuously recorded their times throughout the entire data collection period (June 1 to August 24; 85 days), and the forms were collected every other week. The time values were recorded on a master spreadsheet. 
This single copy is for your personal, non-commercial use only.

For permission to reprint multiple copies or to order presentation-ready copies for distribution, contact CJHP at cjhpedit@cshp.ca

At the end of the data collection period, the proportion of NF medication orders processed through each path of the assessment algorithm was calculated. At that point, any NF medication orders designated as "medication not available" in the dispensary were considered as patients' own medications, and orders not yet resolved by clinical pharmacists were considered as having been dispensed from hospital supply, since they would likely have been processed as such if data collection had continued. The times spent on each dispensary and clinical task were calculated, and the average time was calculated. The average time required to assess a typical NF medication order was calculated by summing the time for each algorithm path, weighted by the proportion of orders passing through each path. The average time for NF medication orders dispensed using patients' own medications or hospital supply was similarly calculated, using the weighted-average time for only these 2 paths.

Descriptive statistics were used to report outcomes. Summary statistics were calculated using Microsoft Excel (Microsoft Corp, Redmond, Washington). The amount of time spent on each stage of the NF medication order process was entered into the spreadsheet, and the average total amount of time required for each type of process was then calculated and tabulated.

\section{RESULTS}

During the 15 days of data collection, 341 orders for NF medications were processed. Table 1 shows the average proportions of NF medication orders received in a day that were processed through each path. The most common path was automatic substitution; the least common path was dispensing the order using hospital supply.

The average times required by each path in the NF medication assessment algorithm are presented in Table 2. The quickest way to process an NF medication order was automatically substituting with a formulary alternative. Processing the NF medication order through the use of patients' own medications and doing so through hospital supply were the most timeconsuming paths.

The pharmacy department typically received 22.7 NF medication orders per day. When the proportion of different algorithm paths and the average time for each path were considered, a typical NF medication order required 8:38 min. When the analysis was limited to NF medication orders dispensed using patients' own medications or hospital supply, the average time was $23: 39 \mathrm{~min}$.

\section{DISCUSSION}

In this study, processing a typical NF medication order required about $8 \mathrm{~min}$, depending on the path that the order took through the NF assessment algorithm. Given 22.7 NF
Table 1. Disposition of Nonformulary (NF) Orders Received on a Typical Day*

\begin{tabular}{lrr} 
Algorithm Path & \multicolumn{2}{c}{$\begin{array}{c}\text { Average No. (and \%) } \\
\text { of NF Orders per Day }\end{array}$} \\
\hline Automatic substitution & 13.05 & $(57.4)$ \\
Discontinuation & 1.60 & $(7.0)$ \\
Formulary alternative & 2.75 & $(12.1)$ \\
Patient's own medication & 4.67 & $(20.5)$ \\
Hospital supply & 0.66 & $(2.9)$ \\
Total & 22.73 & \\
\hline *Data were collected on a total of 15 days over the study period.
\end{tabular}

Table 2. Average Time Spent on a Nonformulary Order

\begin{tabular}{lc} 
Outcome & $\begin{array}{c}\text { Time Spent } \\
\text { (minutes:seconds) }\end{array}$ \\
\hline Automatic substitution & $1: 56$ \\
Discontinuation & $15: 42$ \\
Formulary alternative & $12: 58$ \\
Patient's own medication & $19: 18$ \\
Hospital supply & $30: 31$ \\
\hline
\end{tabular}

orders expected daily at $\mathrm{SPH}$, more than $3 \mathrm{~h}$ each day was dedicated by pharmacy staff to NF medication orders.

These times are within the expected range, based on previous studies. Our group recently conducted an unpublished evaluation to measure the time spent on processing only NF orders dispensed using patients' own medications at Burnaby Hospital and Surrey Memorial Hospital. The average times required for processing an NF order dispensed with patients' own medications were 20:54 min and 24:42 min at Burnaby Hospital and Surrey Memorial Hospital, respectively. These times are longer than the time required for a typical order in the current study $(8: 38 \mathrm{~min})$, but similar to the time required specifically for orders dispensed using patients' own medications (19:18 min). In a US study based on interview data, processing NF orders of all types required 4 to $69 \mathrm{~min},{ }^{3}$ which reflects a range similar to the results presented here.

We directly measured the time for tasks pertaining to all NF medication orders in the dispensary, which has not previously been reported in the literature. Similar studies have relied on surveys of pharmacy staff. The method of measurement used in the current study allowed collection of more accurate data. The timing process was meticulous: only relevant tasks in the NF medication order process were considered, and extraneous tasks were disregarded. The response rate from both dispensary and clinical pharmacists allowed for a pool of 341 individual medication orders to be examined. The methods used in this evaluation could be applied to other sites, allowing those with different NF medication procedures to measure their own times.

One limitation of this study was that medication orders were not followed from beginning to end. This extent of data 
collection was not feasible because a single order may be processed by multiple staff over several days, which was beyond the resources available. Instead, separate steps were timed, and the measurements from different orders were averaged and combined to estimate the average time required for a typical NF medication order. One implication of this limitation is that we were unable to directly compare the difference in workload between formulary and NF orders. Instead, we compared the workflow processes for formulary and NF orders and focused on the tasks that were required only for NF orders. Our assumption was that the time for these tasks represented the difference in time required for NF orders, over and above the time required for formulary orders. However, variations in individual orders undoubtedly occurred; for example, some orders for formulary medications might require clarification or interpretation as well.

Because it was not possible to directly observe multiple staff members simultaneously, the clinical pharmacists were asked to document their own times, which resulted in estimated values for time spent on NF orders outside the dispensary. Another limitation was relying on dispensary pharmacists to record NF medication orders received each day on the data collection form. During busy periods in the dispensary, staff members occasionally forgot to record orders. As a result, this study likely underestimates the total number of NF medication orders each day.

Future studies should include objective measurements of the time required for clinical pharmacists to complete tasks related to NF medication orders. Ideally, an observer would be assigned to one specific clinical pharmacist for a series of shifts. If more resources were allocated to data collection, a team of investigators could follow multiple staff members to capture all aspects of NF orders from beginning to end. An analysis of the NF medications ordered should also be performed, as differences between medications may be associated with variations in time requirements.

Supplying medications through patients' own medications and pharmacy stock may take several days, as the medications need to be either brought in or delivered to the hospital. For some patients, delays in therapy may negatively affect health outcomes. Future studies should investigate possible associations between delays in supplying NF medications and clinically significant patient outcomes.

In this study, the typical NF medication order required 8:38 min for dispensary and clinical staff to assess and process. However, the times associated with NF orders depended on how each order was ultimately dispensed: automatic substitutions required less than $2 \mathrm{~min}$, whereas orders dispensed using patients' own medications or the hospital supply required $20 \mathrm{~min}$ or more.

The time required to process NF medication orders can be used to inform formulary decisions when a given medication is no better or worse (in terms of efficacy and safety) than current options. Currently, the budget impact analyses included in medication reviews conducted for formulary decisions may account for only the costs of medication supply. These analyses can be oversimplistic and favour leaving newer, more expensive medications off the formulary. However, time spent performing tasks related to these NF orders takes pharmacists away from other direct patient care activities. As such, the labour costs of processing NF medication orders may not be worth the amount saved by not listing the medication on formulary. For example, in Lower Mainland Pharmacy Services, where the median hourly salary is $\$ 49.11$ (2016 dollars), ${ }^{6}$ the estimated labour cost-equivalent for a typical NF order is $\$ 7.07$. This information can be used to set a threshold whereby a medication that is equivalent in safety and efficacy to formulary alternatives will cost more as an NF medication than it would cost if listed on formulary. To illustrate, in a simplistic scenario where an NF medication needs to be assessed 100 times in a year, the savings generated by leaving this medication off the formulary should exceed $\$ 707$ to justify the decision on financial grounds alone. Therefore, when the labour costs of assessing NF medication orders are included in determining workload cost equivalence, the true cost of formulary decisions can be evaluated.

\section{References}

1. Chase KA. Medication management. In: Halford DA, Brown TR, editors. Introduction to hospital and health-system pharmacy practice. Bethesda (MD): American Society of Health-System Pharmacists; 2010. p. 59-80.

2. Non-formulary and excluded drugs_LMPS. In: Pharmacy services policy manual. Vancouver (BC): Lower Mainland Pharmacy Services; 2015.

3. Helmons PJ, Kosterink JGW, Daniels CE. Formulary compliance and pharmacy labor costs associated with systematic formulary management strategy. Am J Health Syst Pharm. 2014;71(5):407-15.

4. Wong GYC. Cost impact of using patients' own multidose medications in hospital. Can J Hosp Pharm. 2014;67(1):9-16.

5. St. Paul's Hospital: locations and services. Vancouver (BC): Vancouver Coastal Health; 2014 [cited 2016 Jun 27]. Available from: www.vch.ca/locations-andservices/find-locations/?site_id=784

6. Wage schedules. In: $2014-2019$ Health Science Professionals Bargaining Association collective agreement. New Westminster (BC): Health Sciences Association; 2016.

Elaine Chang is a candidate for a BSc(Pharm) degree in the Faculty of Pharmaceutical Sciences, The University of British Columbia, Vancouver, British Columbia.

Angus Kinkade, BSC(Pharm), ACPR, PharmD, MSC, is with Lower Mainland Pharmacy Services and Vancouver General Hospital, Vancouver, British Columbia.

Anthony C H Tung, BSC(Pharm), ACPR, MBA, is with Lower Mainland Pharmacy Services, Surrey, British Columbia.

Aaron M Tejani, BSc(Pharm), PharmD, is with Lower Mainland Pharmacy Services, Vancouver, British Columbia.

Competing interests: For activities not directly related to the topic of this article, Aaron Tejani has received a grant from the College of Pharmacists of British Columbia and payment for lectures on a variety of topics from the University of British Columbia, Vancouver Island 
Health Authority Pharmacy Services, and various professional societies and organizations; he also serves on the UBC Therapeutics Initiative. No other competing interests were declared.

\section{Address correspondence to:}

\section{Dr Angus Kinkade}

Lower Mainland Pharmacy Services

Vancouver General Hospital

Heather Pavilion, Level D

2733 Heather Street

Vancouver BC V5Z $1 \mathrm{Mg}$ e-mail: angus.kinkade@fraserhealth.ca

Funding: None received.

Acknowledgements: The authors would like to thank Linda Tang and Angela Lo for their help in planning the project, Taylor Quon for his work on previous iterations of this project, and the staff at the St Paul's Hospital Pharmacy Department for their dedicated support in collecting data.

\section{BEST.mis better}

\section{One resource for all types of compounding by pharmacies}

\section{WHAT'S INSIDE?}

- Information for pharmacists, pharmacy technicians, planners, architects, engineers-and others who are involved in decisions or activities that affect compounding

- Guidelines for aseptic compounding, non-aseptic compounding, and compounding which involves hazardous drugs-including radiopharmaceuticals

- Best and leading guidelines on topics such as training, planning and designing the physical environment, developing an air quality strategy, cleaning and decontaminating areas, monitoring the environment, garbing and hand hygiene, developing compounding procedures, documenting, and much more-all in only 230 pages

\section{Learn what best looks like: add \\ this publication to your library!}

HAVE A SNEAK PEEK OR ORDER AT: www.cshp.ca/compounding CSHP MEMBERS PAY A DISCOUNTED PRICE
Canadian Society of Hospital Pharmacists
Société canadienne des pharmaciens d'hôpitaux 